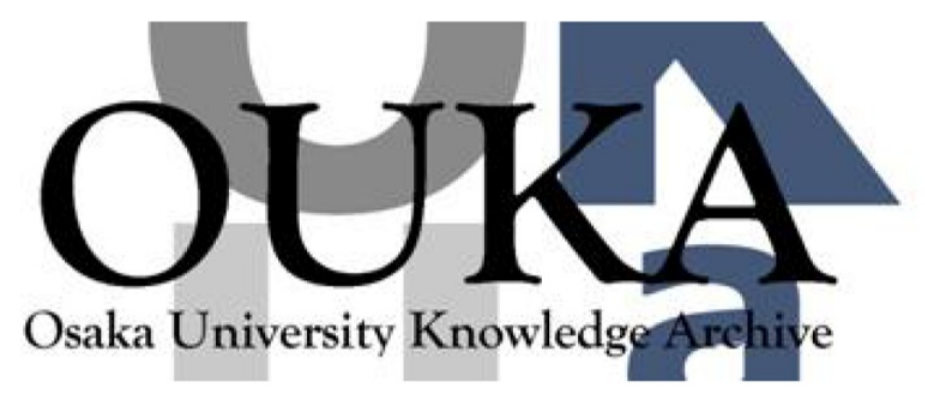

\begin{tabular}{|c|l|}
\hline Title & Review of progress in Fast Ignition \\
\hline Author(s) & Tabak, M.; Clark, D. S. ; Hatchett, S.P. et al. \\
\hline Citation & Physics of Plasmas. 12(5) p. 057305 \\
\hline Issue Date & $2005-05$ \\
\hline oaire:version VoR \\
\hline URL & https://hdl. handle. net/11094/3277 \\
\hline rights & \\
\hline Note & \\
\hline
\end{tabular}

Osaka University Knowledge Archive : OUKA

https://ir. Library. osaka-u. ac. jp/

Osaka University 


\title{
Review of progress in Fast Ignition ${ }^{a)}$
}

\author{
M. Tabak, ${ }^{\text {b) }}$ D. S. Clark, S. P. Hatchett, M. H. Key, B. F. Lasinski, R. A. Snavely, \\ S. C. Wilks, and R. P. J. Town \\ Lawrence Livermore National Laboratory, P. O. Box 808, Livermore, California 94550 \\ R. Stephens and E. M. Campbell \\ General Atomics, P. O. Box 85608, San Diego, California 92121-1122 \\ R. Kodama, K. Mima, and K. A. Tanaka \\ Institute of Laser Engineering, Osaka University, 2-6 Yamada-oka, Suita, Osaka, Japan 565-0871 \\ S. Atzeni \\ Dipartimento di Energetica, Università di Roma, "La Sapienza," and INFM, Via A. Scarpa, \\ 1400161 Roma, Italy \\ R. Freeman \\ 425 Stillman Hall, The Ohio State University, Columbus, Ohio 32210-1123
}

(Received 15 November 2004; accepted 20 December 2004; published online 5 May 2005)

\begin{abstract}
Marshall Rosenbluth's extensive contributions included seminal analysis of the physics of the laser-plasma interaction and review and advocacy of the inertial fusion program. Over the last decade he avidly followed the efforts of many scientists around the world who have studied Fast Ignition, an alternate form of inertial fusion. In this scheme, the fuel is first compressed by a conventional inertial confinement fusion driver and then ignited by a short $(\sim 10 \mathrm{ps})$ pulse, high-power laser. Due to technological advances, such short-pulse lasers can focus power equivalent to that produced by the hydrodynamic stagnation of conventional inertial fusion capsules. This review will discuss the ignition requirements and gain curves starting from simple models and then describe how these are modified, as more detailed physics understanding is included. The critical design issues revolve around two questions: How can the compressed fuel be efficiently assembled? And how can power from the driver be delivered efficiently to the ignition region? Schemes to shorten the distance between the critical surface where the ignitor laser energy is nominally deposited and the ignition region will de discussed. The current status of Fast Ignition research is compared with our requirements for success. Future research directions will also be outlined. (C) 2005 American Institute of Physics. [DOI: 10.1063/1.1871246]
\end{abstract}

\section{INTRODUCTION}

Fast Ignition ${ }^{1}$ is a form of inertial fusion in which the ignition step and the compression step are separate processes. The invention of chirped pulse amplification ${ }^{2}$ of lasers spurred research in this area because these lasers can, in principle, supply energy to the fusion ignition region as fast as the convergence of stagnating flows can for the conventional ignition scheme. In the original concept, the delivery of this ignition laser energy is mediated by the transport of relativistic electrons produced in the laser-plasma interaction. Another variant of this scheme uses protons ${ }^{3}$ driven by these fast electrons to deliver the energy to the fuel. Fast Ignition offers the possibility of higher gains, lower driver energy and cost required to achieve economically interesting gains, flexibility in compression drivers (lasers, pulsed power, and heavy ion beam accelerators), innovative reactor chamber concepts, and lower susceptibility to the effects of hydrodynamic mix than the conventional inertial fusion scheme. Researchers around the world have studied this fu-

\footnotetext{
a) Paper HI1 6, Bull. Am. Phys. Soc. 49, 175 (2004).

${ }^{b)}$ Invited speaker.
}

sion scheme intensively for the past dozen years. The challenging physics and opportunities motivated Marshall Rosenbluth to encourage and to contribute to Fast Ignition research. This report will review the Fast Ignition scheme, the progress made over the past decade, and possible directions for the future. The plan of this paper is as follows: Section II describes ignition requirements and a simple gain model. Section III describes a typical implosion used to assemble fuel, its consequences for Fast Ignition, and how this implosion might be modified. Section IV presents results on the coupling of high-intensity laser light and plasmas, the generation of fast electrons, and the subsequent transport of these electrons. Section V describes various techniques to improve the efficiency of the transport of energy between the nominal critical surface where the ignitor laser energy is deposited and the compressed fuel where ignition occurs. Section VI summarizes ${ }^{4}$ and concludes this paper.

\section{IGNITION REQUIREMENTS AND GAIN MODELS}

Ignition requires that a sufficiently large region of fusion fuel be heated to the ignition temperature. This ignition temperature depends on the ignition region size given by its 

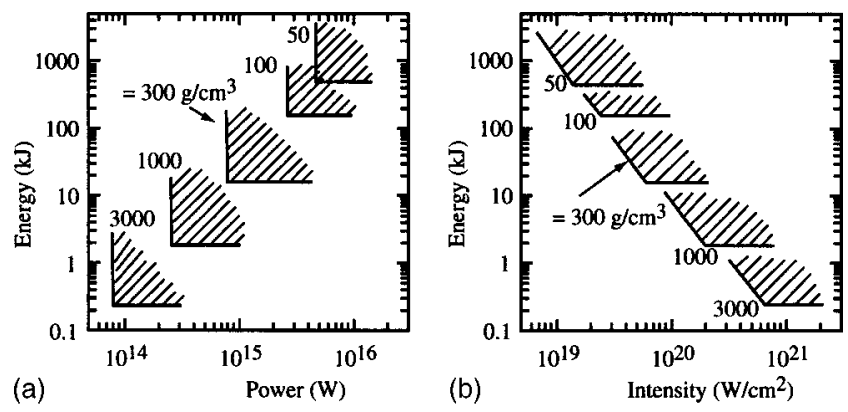

FIG. 1. (a) Isochoric ignition window in energy-power space for a variety of compressed fuel densities; (b) ignition windows in energy-intensity space.

column density, $h=\int \rho d R \approx \rho R$. The hotspot energy requirement in megajoules is then: $E_{\mathrm{ign}}=144[(\bar{Z}+1) / \bar{A}] M T$, where $M=4 \pi / 3(\rho R)^{3} / \rho^{2}$ and $\rho$ is given in $\mathrm{g} / \mathrm{cm}^{3}, T$ in $\mathrm{keV} . \bar{Z}, \bar{A}$ are the average atomic number and atomic weight, respectively. For an equimolar deuterium-tritium (D-T) plasma $\bar{Z}$ $=1$ and $\bar{A}=2.5$. In conventional inertial fusion, a low density hotspot and ignition region is surrounded by a relatively cold and dense main fuel region, where the bulk of the yield is produced. The heating of the hotspot and the compression of the main fuel to high density happen simultaneously as the kinetic energy of the imploding shell is converted into internal energy of compressed fuel during stagnation. Because the hotspot and the main fuel are sonically connected, their pressures are approximately equal ( $200 \mathrm{Gbar})$. The minimum ignition requirements (in terms of $T$ and $h$ ) depend on how well energy is confined in the hotspot. The losses can include radiation, electron conduction, and hydrodynamic work. In an isobaric configuration, the hotspot is tamped and its hydrodynamic losses are limited during ignition. In addition, some self-heating during the implosion reduces the energy that must be delivered to the hotspot for ignition. In contrast, in the isochoric configuration used in Fast Ignition, the ignition region is far out of pressure balance with the surrounding fuel, so hydrodynamic losses can be significant.

Atzeni and collaborators ${ }^{5}$ by performing a series of twodimensional (2-D) simulations where energy is injected into precompressed D-T fuel found that the ignition energy in an isochoric configuration was approximately 5 times greater than that for isobaric ignition. This corresponds to $h$ $=0.6 \mathrm{gm} / \mathrm{cm}^{2}$ and $T=12 \mathrm{keV}$. Figure 1 shows the ignition windows in energy, power, and intensity for various fuel densities. The minima for these quantities, for deposition ranges between 0.3 and $1.2 \mathrm{~g} / \mathrm{cm}^{2}$, can be parametrized as functions of $\rho$ :

$$
\begin{aligned}
& E_{\text {ign }}(\mathrm{kJ})=140\left(\frac{\rho}{100 \mathrm{~g} / \mathrm{cm}^{3}}\right)^{-1.85}, \\
& W_{\text {ign }}(\mathrm{W})=2.6 \times 10^{15}\left(\frac{\rho}{100 \mathrm{~g} / \mathrm{cm}^{3}}\right)^{-1},
\end{aligned}
$$

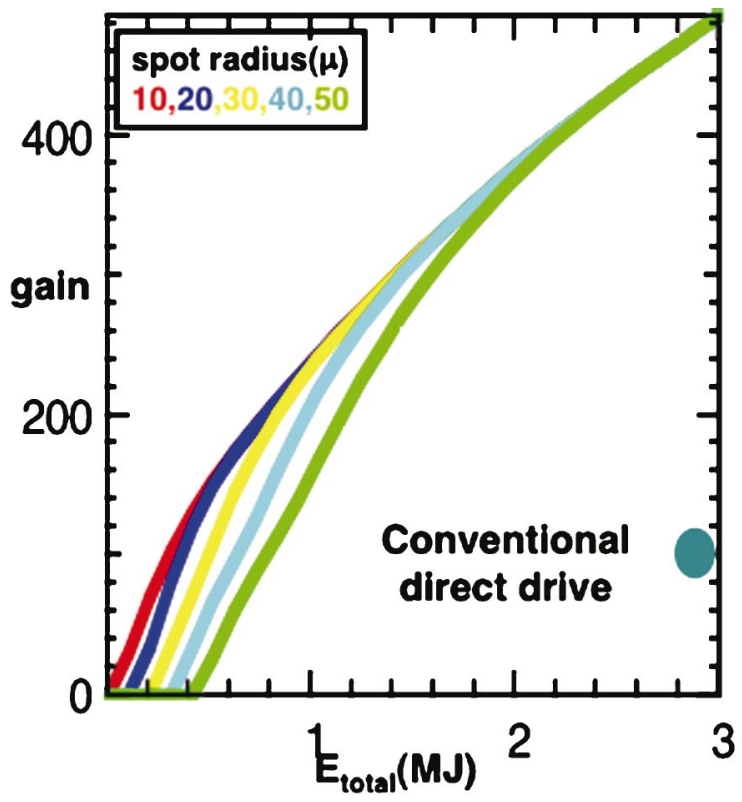

FIG. 2. (Color). Gain vs total laser energy for capsules directly imploded with $0.35 \mu \mathrm{m}$ lasers for a variety of ignition energy deposition radii. The model assumes $25 \%$ coupling from ignition laser to compressed fuel.

$$
I_{\text {ign }}\left(\mathrm{W} / \mathrm{cm}^{2}\right)=2.4 \times 10^{19}\left(\frac{\rho}{100 \mathrm{~g} / \mathrm{cm}^{3}}\right)^{0.95} .
$$

The coupling efficiency will determine the ignitor laser requirements. Preliminary results show that if the ignition energy is used to drive an ultrahigh pressure reimplosion of the compressed ignition region instead of directly heating it, the ignition energy can be reduced by at least a factor of 2 with an associated increase in spot size and delivery time. ${ }^{6}$

By combining these ignition requirements with models of directly driven implosions ${ }^{7,8}$ that supply the hydrodynamic efficiency, $\eta_{\text {hyd }}$; the relation between ignition laser intensity and the temperature ${ }^{9}$ of the hot electron distribution:

$$
T(\mathrm{MeV})=\left(\frac{I_{\text {ign-laser }}}{1.2 \times 10^{19}\left(\mathrm{~W} / \mathrm{cm}^{2}\right)}\right)^{1 / 2}
$$

for an ignition laser with wavelength $1.05 \mu \mathrm{m}$; and the particle range, $R\left(\mathrm{~g} / \mathrm{cm}^{2}\right)=0.6 T(\mathrm{MeV})$, we can derive model gain curves. ${ }^{10}$ The nominal model assumes a $25 \%$ coupling efficiency, $\eta_{\text {ign }}$, from the ignition laser to the fuel with duration and spot size inferred from the ignition requirements given above. We also take the fuel to be Fermi-degenerate during the implosion, although entropy will be generated during stagnation leading to a higher fuel adiabat. Using cones and/or proton beams to deliver energy to the ignition region (see below) breaks the correlation among the ignition intensity, the laser intensity and the particle deposition range, because the laser energy is deposited over a larger area and then concentrated into the ignition spot. Direct illumination produces particles with the longest ranges and hence the largest ignition requirements. Figure 2 shows the dependence of the gain curve on the minimum laser spot size. Note that the nominal model produces gain 100 at about $10 \%$ of the 
TABLE I. Total laser energy required for gain 100.

\begin{tabular}{lc}
\hline \hline Parameter & $E_{\text {laser }}(\mathrm{MJ})$ \\
\hline Nominal model & 0.3 \\
$E_{\text {ign }} \times 1 / 2$ & 0.1 \\
$\eta_{\text {ign }} \times 0.25$ & 1.7 \\
$\eta_{\text {hydro }} \times 0.5$ & 0.95 \\
range $_{\text {ign }} \times 3$ & 0.75 \\
$0.5 \mu$ m drive & 0.55 \\
\hline
\end{tabular}

energy required from a conventionally ignited directly driven capsule. Table I shows examples of the sensitivity of the gain to various changes in model assumptions including utilizing a $0.5 \mu \mathrm{m}$ wavelength laser as the implosion driver rather than the nominal $1 / 3 \mu \mathrm{m}$ laser. All of these variations show gain 100 occurring with a lower laser energy requirement than conventionally ignited capsules.

\section{IMPLOSION RESULTS}

The Fast Ignition concept requires an implosion to assemble the fuel into a compact and dense mass. Typical inertial confinement fusion implosions are designed to produce a central high entropy region where ignition occurs. Figure 3(a) shows density profiles produced during various moments of a typical direct drive implosion. There are two salient features: the critical density (above which the laser cannot propagate) is located almost a millimeter from the high density region and the compressed fuel assembles into a high density shell surrounding a central region with $10 \%$ of the peak density. This thin shell will be difficult to ignite by injecting heat because it can disassemble in two directions during the ignition phase. In addition, the burn efficiency of the fuel, once ignited, is reduced because its column density $\left(\int \rho d R\right)$ is smaller when distributed as a shell than as a uniform sphere of the same mass. We can eliminate the central low density region in several ways: (1) introduce a high- $Z$ seed (e.g., Xe with $3 \times 10^{-5}$ atomic fraction) into the center and radiate away the entropy [see Fig. 3(b)]. For the implosion shown in Fig. 3, this a 10\% energy cost. (2) Expel the central gas through openings (either preformed or produced during the implosion) in the shell. (We discuss this below.) Or (3) design the implosion so that with proper pulseshaping, a shell can be imploded to form a uniform sphere. Figure 3(c) shows a sequence of snapshots in time of the self-similar implosion of a shell, driven by pressure applied to its outer surface, that upon stagnation becomes a uniform sphere. Producing the initial state shown in the figure from a uniform shell at rest will require a sequence of well-timed shocks and remains to be accomplished. The final design will be ablation driven and hence will also have an extensive coronal plasma surrounding the compressed fuel.

\section{LASER COUPLING TO FAST ELECTRONS AND SUBSEQUENT TRANSPORT}

The generation of the ignitor electrons is accomplished through the absorption of the laser via collisionless
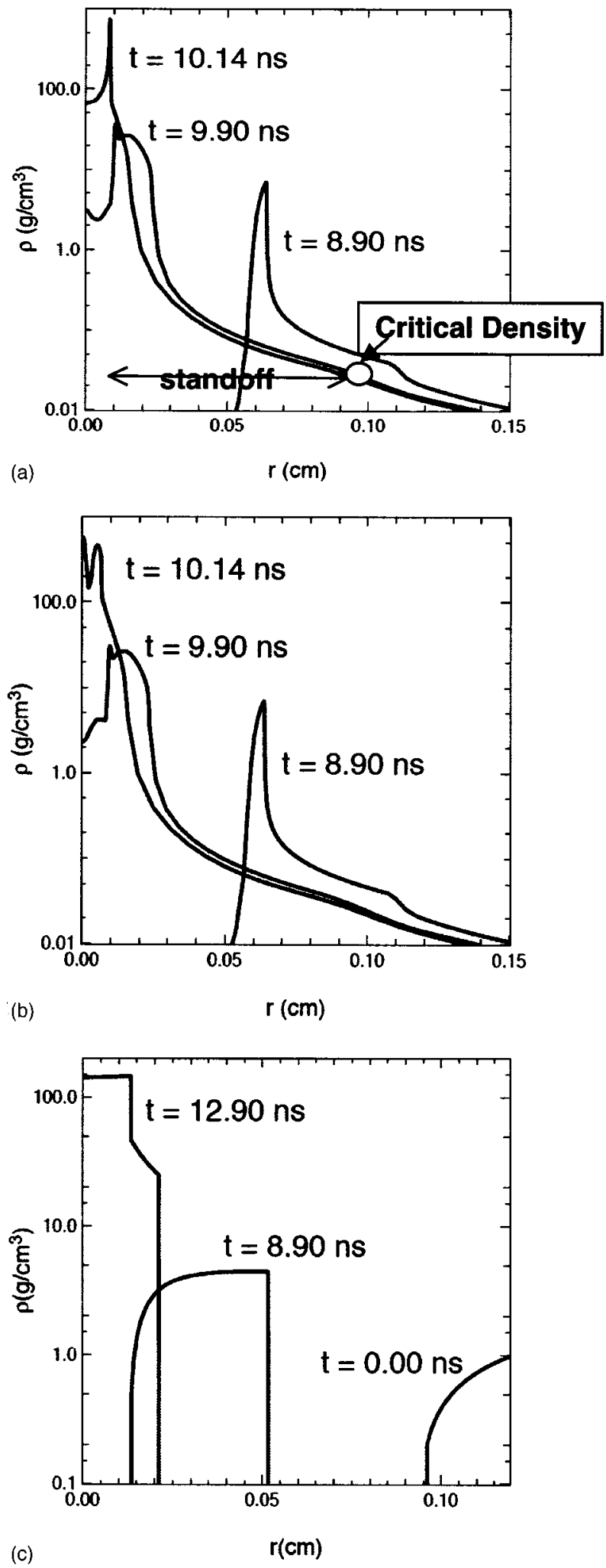

FIG. 3. (a) Snapshots of $\rho$ vs $r$ for directly driven implosion with critical surface marked; (b) like (a) but central fuel has Xe introduced into central gas; (c) snapshots of self-similar implosion taking shell into uniform sphere.

mechanisms, ${ }^{11}$ such as resonance absorption, $J \times B$ heating, and Raman scattering. Figure 4 shows the conversion efficiency of intense light to forward-going relativistic electrons. $^{12}$ These data were inferred from experiments 


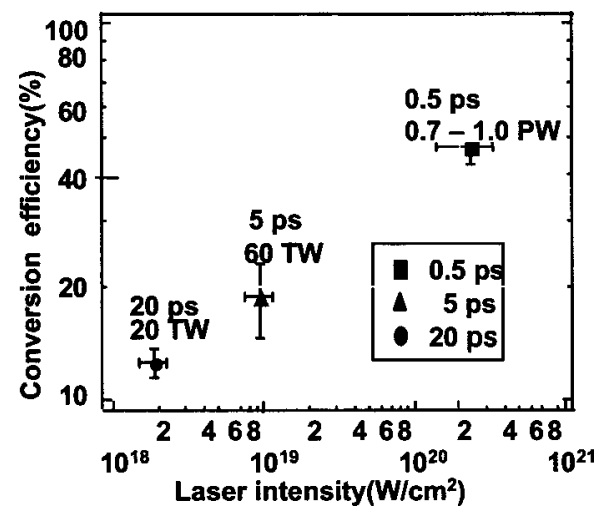

FIG. 4. Conversion efficiency into forward going electrons as a function of laser intensity for $1 \mu \mathrm{m}$ light.

where targets composed of varying thicknesses of aluminum followed by $50 \mu \mathrm{m}$ molydenum $K_{\alpha}$ fluor layers and then 2-mm-thick equimolar carbon-hydrogren $(\mathrm{CH})$ beam stops were illuminated by intense laser light. The $K_{\alpha}$ signals were then compared to those obtained by ITS, ${ }^{13}$ a Monte Carlo particle tracking code, using Maxwellian distributions for the injected electrons. Because this analysis did not include the self-consistent electric and magnetic fields, the quoted results represent lower estimates for the coupling efficiencies.

Physics at multiple scales affects the transport of the intense relativistic electron beams produced by the laser. For Fast Ignition applications the beam electron temperature is in the range $0.5-3 \mathrm{MeV}$, the forward current is a giga-ampere and the forward current density, $\mathbf{j}$, is about $10^{14} \mathrm{~A} / \mathrm{cm}^{2}$. This current leads to large space-charge and magnetically induced electric fields that draw a return current approximately equal to the forward current. The return current is composed, in part, of low energy electrons. Scattering of these returning electrons produces a resistive $\mathbf{E}$ field $=\mathbf{j} / \sigma \sim 10^{8} \mathrm{~V} / \mathrm{cm}$ in aluminum below $100 \mathrm{eV}$ temperature, where $\sigma$ is the conductivity. For existing experiments in aluminum or $\mathrm{CH}$, Joule heating produced by the return current dominates the heating of the background plasma. In the Fast Ignition regime, where the fuel has been compressed to a density of hundreds of $\mathrm{g} / \mathrm{cm}^{3}$, the temperature is $10-100$ times larger and $Z$, the atomic number, equals unity, Joule heating is unimportant. Because the current densities are so large, coherent scattering ${ }^{14}$ of pairs of relativistic electrons off background electrons and ions may increase stopping and multiple scattering relative to incoherent single particle scattering. ${ }^{15}$

On the scale of the collisionless plasma skin depth $(0.01-10 \mu \mathrm{m})$ the collionless and collisional version of the filamentation instability for cold beams have growth rates that scale like $\alpha^{1 / 2} \omega_{e}$; where $\alpha=n_{b} / n_{e}, n_{e}$ is the background plasma density, $n_{b}$ is the beam plasma density and $\omega_{e}$ is the background plasma frequency. Finite transverse beam temperature, $T$, and reduced values of $\alpha$ lead to reduced linear growth rates. ${ }^{16}$ Silva et al. ${ }^{16}$ found, using a waterbag beam distribution, that there is (Fig. 5) a threshold for instability growth that depends on $\alpha$ and $T$. Large values of $T / E_{\text {beam, }}$ where $E_{\text {beam }}$ is the beam particle kinetic energy, have been inferred from measurements of bremsstrahlung radiation pro-

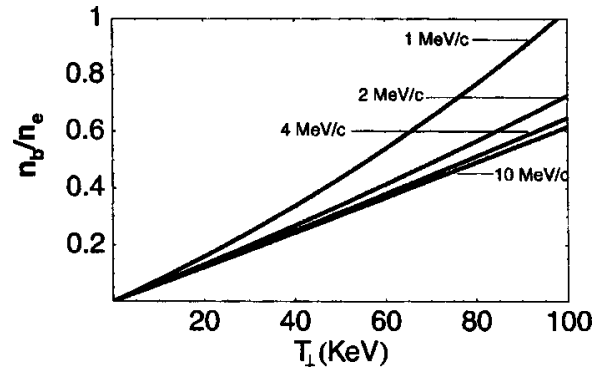

FIG. 5. Instability boundaries in the space of beam transverse temperature and ratio of beam to background density for the Weibel instability for various electron beam kinetic energies.

duced during illuminations of high- $Z$ targets by intense laser beams. The radiation was distributed within a cone half angle of $1 \mathrm{rad}$ about the beam centroid, much more than would be produced by multiple scattering off nuclei. ${ }^{17}$ This large apparent $T / E_{\text {beam }}$ may be a result of the electron acceleration process in the laser-plasma interaction as seen in particle-incell (PIC) code calculations or may be produced as the instability evolves. For typical experimental conditions this graph implies that there will be no growth of the collisionless filamentation instability. However, finite background plasma resistivity and more general beam distributions allow growth below the thresholds plotted in Fig. 5. In addition, for Fast Ignition $\alpha$ ranges from 1 (at the critical surface) to $10^{-5}$ in the ignition region. For sufficiently gentle plasma density profiles, there is a window where this instability can grow.

Figure $6^{18}$ shows idealized 2-D PIC predictions of the scaled energy loss during the nonlinear phase for cold monoenergetic relativistic electron beams as they propagate through background collisionless plasmas with $\alpha$ varying from 0.1 to 0.02 . When $\alpha=n_{b} / n_{e}=0.1$, the energy loss rate corresponds to stopping in a range of $5 \times 10^{-5} \mathrm{~g} / \mathrm{cm}^{2}$, a stopping power $10^{4}$ larger than classical. The stopping power decreases as $\alpha$ decreases. Magnetic trapping causes the saturated magnetic field value to depend on the linear growth rate. ${ }^{16}$ Winding the electron paths by magnetic deflection will also increase the apparent stopping power due to collisions.

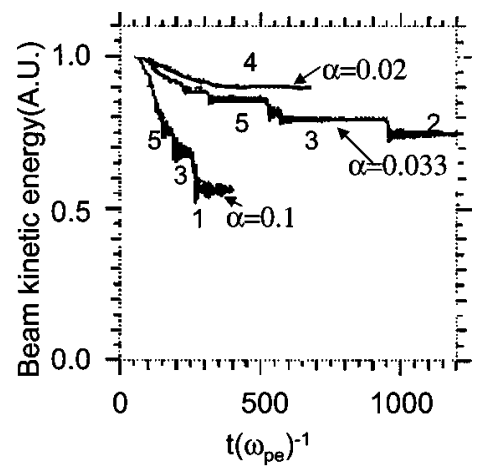

FIG. 6. Fraction of incident beam energy as a function of time in units of inverse plasma frequency for three ratios of plasma to beam particle density: $[50,30,10]$. The highest ratio corresponds to the uppermost curve. The numbers on each curve are the number of filaments at any given time. 


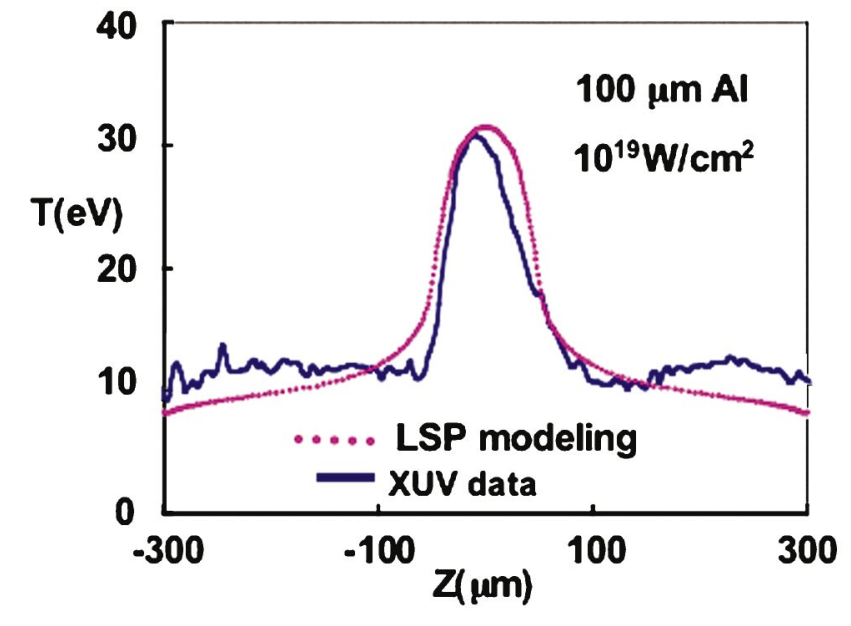

FIG. 7. (Color). Temperature measured (black) at rear surface of $100 \mu \mathrm{m} \mathrm{Al}$ slab when irradiated with $100 \mathrm{~J}$ of laser light with peak intensity $10^{19} \mathrm{~W} / \mathrm{cm}^{2}$ together with LSP simulation of the experiment.

However, it appears that such extreme anomalous stopping is not required to explain existing experiments. Figure 7 shows the temperature measured at the rear surface of a 100$\mu \mathrm{m}$-thick aluminum $\operatorname{slab}^{19}$ together with the results of a simulation $^{20}$ performed with the hybrid, collisional PIC code, LSP. $^{21}$ The simulation used the laser intensity pattern, phenomenological coupling efficiencies, and the $\mathrm{Beg}^{22}$ relation between the laser intensity and the average energy of the electrons produced: $E_{\text {hot }}(\mathrm{MeV})=0.1\left(I \lambda^{2} / 10^{17} \mathrm{~W} /\right.$ $\left.\mathrm{cm}^{2} \mu \mathrm{m}^{2}\right)^{1 / 3}$. Electrons were injected into the simulation using an isotropic thermal spread with a temperature of 300 $\mathrm{keV}$ added to a drift energy given by $E_{\text {hot }}$. The resistivity used was approximately that given by Lee and More. ${ }^{23}$ In particular, no subscale anomalous resistivity model was used. The results were fairly insensitive to the grid resolution of the simulation indicating that anomalous stopping by many orders of magnitude did not occur in producing the rear surface pattern and is unlikely to be important for energy deposition in the dense Fast Ignition core.

Beam spreading is a significant issue. The beam spot in Fig. 7, after a $100 \mu \mathrm{m}$ transit, had a $72 \mu \mathrm{m}$ full width at half maximum and would be marginally acceptable. However, if the divergence persisted, the spot size after a $1 \mathrm{~mm}$ transit from the critical surface to the ignition region (as discussed in Sec. III) would lead to a coupling efficiency of less than $1 \%$. Experiments ${ }^{24}$ in solids where embedded $K_{\alpha}$ fluors are imaged show that the beam spreads with a half-angle of $20^{\circ}-$ $25^{\circ}$ starting from a front-surface spot of about 5 times the radius of the nominal laser spot. Also seen spectroscopically was that a front layer, initially a micrometer thick, was heated an order of magnitude more than the bulk plasma and corresponds to less than a $10 \%$ beam energy loss. Both the anomalous front surface heating and the increase of electron beam size above that of the laser spot can have adverse effects on the prospects for Fast Ignition as laser irradiation durations and plasma scale heights increase. Neither is understood at this time and is the subject of continuing research.

\section{SPANNING THE DISTANCE BETWEEN THE CRITICAL SURFACE AND THE IGNITION REGION}

From the previous discussion, it is clear that for electron driven Fast Ignition the distance between the critical surface and the high density region must be minimized. Boring a hole through the plasma; and separating the ablation region from the ignition laser path with a conical divider are schemes that have been proposed to accomplish this. In addition, hot electron driven proton beams can be ballistically focused, deliver energy over a standoff distance and heat the target. We will now briefly review the progress made in these three approaches.

The hole-boring scheme ${ }^{1}$ uses ponderomotive pressure, thermal pressure, as well as the relativistic increase of the critical density $\left(n_{c}\right)$ with laser intensity to shorten the distance between the critical density and the ignition region. Young et al. ${ }^{25}$ have shown $80 \%$ efficiency in the propagation of $60 \mathrm{~J}$ of laser energy through plasmas with peak density $0.3 n_{c}$, scale size $500 \mu \mathrm{m}$ over 500 ps with intensity $5 \times 10^{15} \mathrm{~W} / \mathrm{cm}^{2}$. Recently, Tanaka et al. ${ }^{26}$ have propagated a picosecond pulse of light at $10^{19} \mathrm{~W} / \mathrm{cm}^{2}$ through $10 n_{c}$, with $5 \%$ efficiency, demonstrating the relativistic effect as well as ponderomotive hole boring.

After propagating through a slab, some fraction of the relativistic electrons produced in the laser-plasma interaction at the front surface will escape at the rear surface. This will generate a large electrostatic field that in turn will accelerate ions from the back surface of the foil. Hydrogen constituents of the pump oil adsorbed to back surface of the slab or of a hydrocarbon substrate ${ }^{27}$ are sources for proton beams. Because the incident electron current is so large, the peak fields are of order $10^{9}-10^{10} \mathrm{~V} / \mathrm{cm}$ at the proton front and have duration of a few picoseconds. The efficiency of conversion of laser energy into proton energy as large as $12 \%$ has been measured. ${ }^{28}$ The bulk of the acceleration occurs in a few microns and is one-dimensional to first order. Hence proper shaping of the foil can be used to focus the beam to a small spot. Patel et al., ${ }^{29}$ using the 10J JanUSP ${ }^{30}$ laser, focused a proton beam to a $30-\mu \mathrm{m}$-diam spot from $160 \mu \mathrm{m}$ away and heated the target spot to $23 \mathrm{eV}$. The energy coupled to the target foil was $0.2 \mathrm{~J}$. However, for this to be a path to Fast Ignition a number of issues must be resolved:

(1) Is the proton production efficiency adequate?

(2) Is the proton spectrum produced consistent with optimum deposition depth in the fuel?

(3) How do the efficiency and focusing scale to large energies and pulse lengths?

(4) Will the energy delivery time be consistent with ignition duration?

(5) Will multiple scattering through intervening materials such as hohlraum walls or the tips of cones spread the beam unacceptably?

Cone focus geometry provides an open path for the ignition laser. ${ }^{31}$ Figure 8 (a) illustrates this scheme. If the shell is asymmetrically imploded, the central gas mass is expelled and the shell assembles into a compact mass with little cone mass entrained by the shell. Figure $8(b)^{32}$ shows the result of 


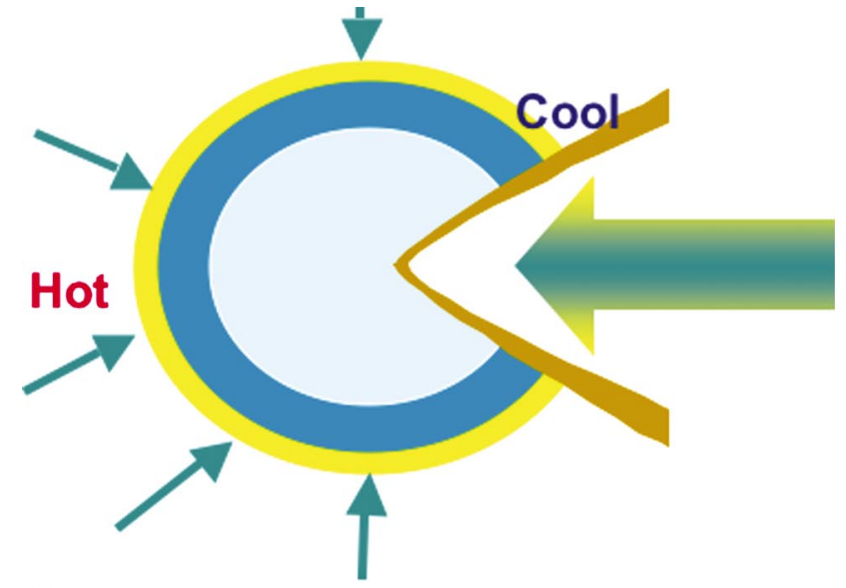

(a)

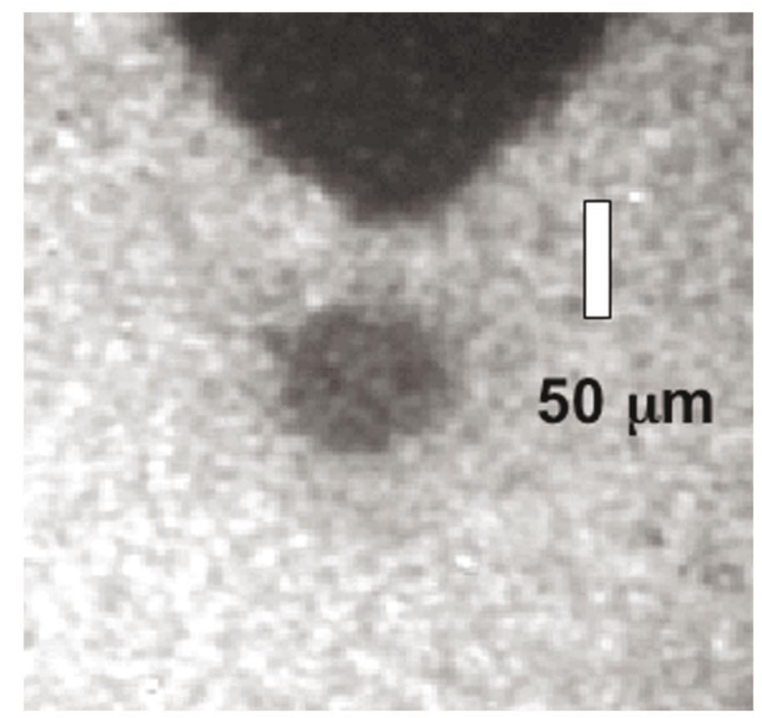

(b)

FIG. 8. (Color). (a) Cartoon of cone-focus target. A shell surrounding an embedded cone is imploded, possibly asymmetrically. The cone provides access for an intense laser beam incident from the right. (b) Radiograph of directly driven target at Omega. The self-emission has been filtered with an iron filter.

such a directly driven implosion when backlit with $6.7 \mathrm{keV}$ photons and filtered with an iron filter to eliminate selfemission. The initial $\mathrm{CH}$ shell was filled with $5 \mathrm{~atm}$ of deuterium $\left(D_{2}\right)$. The compressed core was less than a diameter away from the cone tip.

Three-dimensional PIC simulations ${ }^{33}$ also show that the cone concentrates energy contained in the laser beam and delivers it to the tip of the cone. The light at the cone tip was 20 times as intense as that at the inlet plane. The electrons are confined to a skin depth near the inner edge of the cone by a balance of magnetic pinch forces pushing the electrons toward the cone axis and electrostatic sheath forces pulling the electrons back into the cone. This collisionless PIC calculation covered an extent of $14 \times 9 \times 9 \mu \mathrm{m}^{3}$ for a duration of 50 fs. The plasma density was $5.5 \times 10^{21} / \mathrm{cm}^{3}$. A gold cone (like that used in experiments) when ionized 30 times has an electron density of $1.8 \times 10^{24} / \mathrm{cm}^{3}$. A hybrid PIC simulation ${ }^{34}$ where $2 \mathrm{MeV}$ electrons are uniformly injected

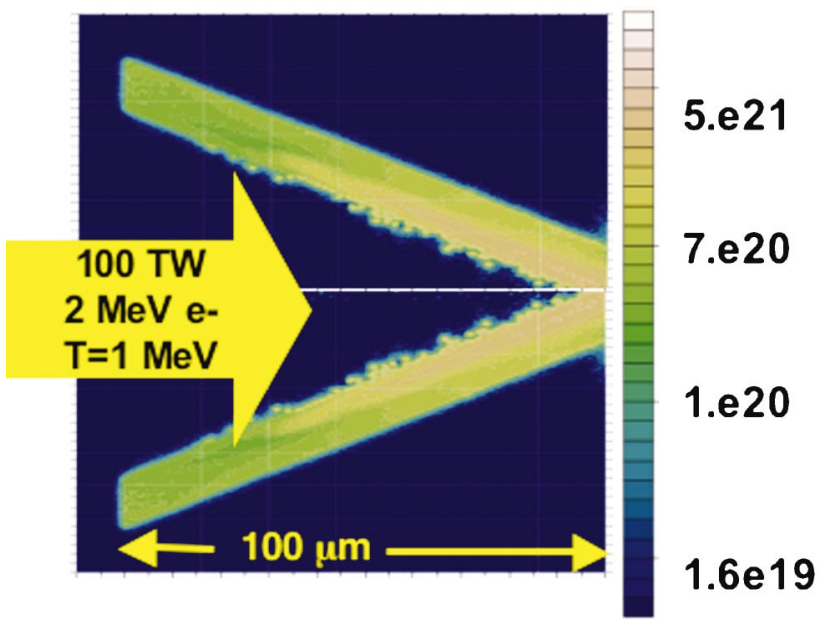

FIG. 9. (Color). Hot electron distribution in gold cone in LSP simulation at 2 ps. $100 \mathrm{TW}$ of electron energy is uniform injected into the inner surface of the cone. The electrons have a drift energy of $2 \mathrm{MeV}$ along the cone axis with a temperature of $1 \mathrm{MeV}$.

parallel to the cone axis with a temperature of $1 \mathrm{MeV}$ into a gold cone with linear dimension $100 \mu \mathrm{m}$ over 2 ps shows electron confinement within a collisional skin depth (Fig. 9). However, the transfer efficiency from surface of the cone to the tip was only about $10 \%$. Multiple scattering of the relativistic electrons in the normal density gold cone folds the path so much that these electrons can only travel $40 \mu \mathrm{m}$ before depositing their energy.

Experiments at Osaka University ${ }^{35}$ measured ultraviolet radiation emitted from the rear surface of slabs with and without gold cones to experimentally examine these issues. A factor of 3 intensity increase was observed when a cone was attached to the front of the slab. Energy collection efficiency will be optimized by varying collector shape, material, and density. Clearly, further research is required.

The most complete Fast Ignition experiments to date have been performed at Osaka University. These experiments ${ }^{36}$ showed that $25 \%$ of incident laser energy were

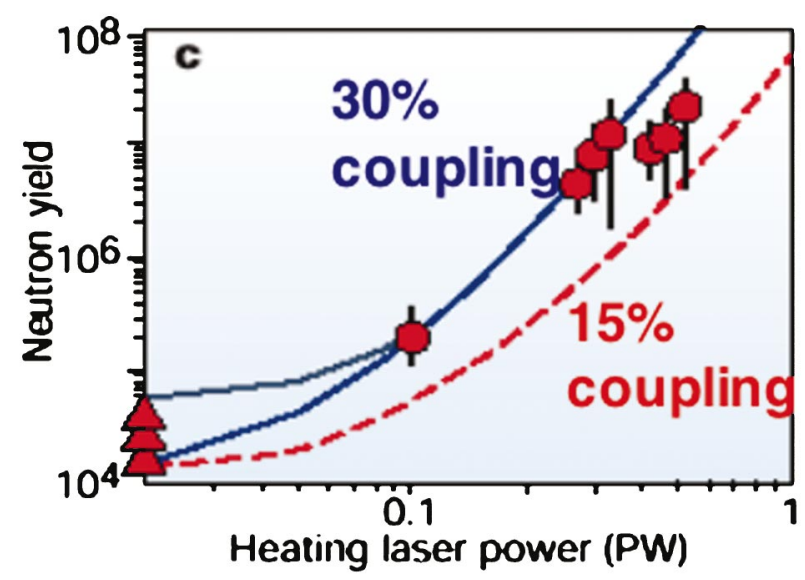

FIG. 10. (Color). Neutron yield in an Osaka cone focus experiment as function of heating laser power. The shell was pre-imploded with $1-2 \mathrm{~kJ}$ of $0.5 \mu \mathrm{m}$ light. 
coupled to a compressed core. A carbon-deuterium (CD) shell was imploded around a cone of gold with $1.2-2 \mathrm{~kJ}$ of $0.53 \mu \mathrm{m}$ light. The shell was compressed to a radius of 15 $\mu \mathrm{m}$ from an initial radius of $250 \mu \mathrm{m}$ achieving a density of $80-100 \mathrm{~g} / \mathrm{cm}^{3}$. This compressed fuel rested on the cone tip. When $3-6 \times 10^{14} \mathrm{~W}$ of laser power was injected down the cone, up to a 1000-fold increase in neutron yield over the yield without laser injection was observed (Fig. 10). When the time of laser injection was varied relative to the time of peak shell compression, the peak neutron yield occurred when the two times coincided. The neutron spectrum corresponded to the deuterium-deuterium (D-D) fusion peak with a thermal width corresponding to an ion temperature of $0.86 \pm 0.1 \mathrm{keV}$. The temperature inferred from the slope of the free-bound continuum slope was $1 \pm 0.1 \mathrm{keV}$. These temperatures correlated well with the neutron yield. If this good coupling can be maintained for larger systems and longer ignitor pulses, then the simple gain model presented in Sec. II would predict high gains with total laser energy of several hundred kilojoules.

\section{SUMMARY}

This paper has reviewed the current status of Fast Ignition research. Fast Ignition involves two stages: First the compressed fuel is assembled and then the fuel is ignited. We have discussed multiple ways to assemble the compressed fuel without a central low-density region which will maximize the target gain. Second, we reviewed laser-electron coupling experiments that showed high 20\%-60\% efficiencies. We examined the physics of the transport of these electrons to the compressed core and reviewed the three principal schemes to improve the transport efficiencies, namely hole boring, proton beams, and cone focus geometry. Currently, the most successful of these is the cone geometry. Integrated gain models show that high gain at sub-megajoule scale can be achieved with the coupling efficiencies demonstrated in recent experiments.

A number of new petawatt laser-implosion system combinations are due to come on line in the years 2007-2008: FIREX $^{37}$ at Osaka University with $10 \mathrm{~kJ}$ of short-pulse laser energy delivered in $10 \mathrm{ps}$; Omega $\mathrm{EP}^{38}$ at the University of Rochester with two $2.5 \mathrm{~kJ}$ beams; $\mathrm{NIF}^{39}$ with $3 \mathrm{~kJ}$, and Z-Beamlet ${ }^{40}$ at Sandia National Laboratory, Albuquerque. These facilities are aimed in part at achieving gain 0.1 and investigating the $1-10 \mathrm{keV}$ material temperature regime.

\section{ACKNOWLEDGMENTS}

This work was performed under the auspices of the U.S. Department of Energy by the University of California, Lawrence Livermore National Laboratory under Contract No. W-7405-ENG-48.

${ }^{1}$ M. Tabak, J. H. Hammer, M. E. Glinsky, W. L. Kruer, S. C. Wilks, J. Woodworth, E. M. Campbell, M. D. Perry, and R. J. Mason, Phys. Plasmas 1, 1626 (1994).

${ }^{2}$ D. Strickland and G. Mourou, Opt. Commun. 55, 447 (1985).

${ }^{3}$ M. Roth, T. E. Cowan, M. H. Key et al., Phys. Rev. Lett. 86, 436 (2001).

${ }^{4}$ Compact disks from the latest Workshops on Fast Ignition will contain material beyond what can be discussed in this review. The 2002 Workshop was held in St. Petes Beach, FL and was organized by M. H. Key (key1@1lnl.gov). The 2004 Workshop was held in Kyoto, Japan and organized by K. A. Tanaka (katanaka@ile.osaka-u.ac.jp)

${ }^{5}$ S. Atzeni, Phys. Plasmas 6, 3316 (1999).

${ }^{6}$ M. Herrmann, S. Hatchett, and M. Tabak, Bull. Am. Phys. Soc. 46, 106 (2001).

${ }^{7}$ J. D. Lindl, Inertial Confinement Fusion. The Quest for Ignition and Energy Gain Using Indirect Drive (Springer, New York, 1998).

${ }^{8}$ S. M. Pollaine and J. D. Lindl, Nucl. Fusion 26, 1719 (1986).

${ }^{9}$ S. C. Wilks, W. L. Kruer, M. Tabak, and A. B. Langdon, Phys. Rev. Lett. 69, 1383 (1993).

${ }^{10}$ M. Tabak, S. Atzeni, E. M. Campbell and K. Tanaka, "Introduction to Fast Ignition," Fusion Sci. Technol. (submitted); M. Tabak and D. A. Callahan, "Models of gain curves for fast ignition," Nucl. Instrum. Methods Phys. Res. A (to be published).

${ }^{11}$ W. L. Kruer, Phys. Plasmas 10, 2709 (2003).

${ }^{12}$ K. Yasuike, M. H. Key, S. P. Hatchett et al., Rev. Sci. Instrum. 72, 1236 (2001).

${ }^{13}$ J. A. Halbleib and T. A. Mehlhorn, Trans. Am. Nucl. Soc. 52, 378 (1986).

${ }^{14}$ C. Deutsch and P. Fromy, Laser Part. Beams 18, 301 (2000).

${ }^{15}$ C. Deutsch, H. Furukawa, K. Mima et al., Phys. Rev. Lett. 77, 2483 (1996).

${ }^{16}$ L. O. Silva, R. A. Fonseca, J. W. Tonge, W. B. Mori, and J. M. Dawson, Phys. Plasmas 9, 2458 (2002); T.-Y. B. Yang, J. Arons, and A. B. Langdon, ibid. 1, 3059 (1994).

${ }^{17}$ S. P. Hatchett, C. G. Brown, T. E. Cowan et al., Phys. Plasmas 7, 2076 (2000).

${ }^{18}$ M. Honda, J. Meyer-ter-Vehn, and A. Pukhov, Phys. Rev. Lett. 85, 2128 (2000); B. F. Lasinski, A. B. Langdon, M. H. Key, and S. P. Hatchett, "Filamentation of relativistic electron beams: Another look," Fourth International Workshop on Laser-Plasma Interaction Physics, Banff, Canada, 21-24 February 2001.

${ }^{19}$ M. H. Key, F. Amiranoff, C. Andersen, S. D. Batani, S. D. Baton, T. Cowan, N. Fisch, R. R. Freeman, L. Gremillet, T. Hall, S. P. Hatchett, J. M. Hill, J. A. King, R Kodama, J. A. Koch, M. Koenig, B. Lasinski, B. Langdon, A. J. MacKinnon, E. Martinolli, P. Norreys, P. Parks, E. PerelliCippo, M. Rabec Le Gloahec, M. Rosenbluth, C. Rousseaux, J. J. Santos, F. Scianitti, R. Snavely, M. Tabak, K. Tanaka, R. P. J. Town, T. Tsutumi, and R. B. Stephens, in Inertial Fusion Sciences and Applications 2003, edited by K. Tanaka, D. D. Meyerhofer, and J. Meyer-ter-Vehn (Elsevier, Paris, 2004), p. 353.

${ }^{20}$ R. P. J. Town, C. Chen, L. A. Cottrill et al., "Simulations of electron transport for Fast Ignition using LSP," Nucl. Instrum. Methods Phys. Res. A (to be published).

${ }^{21}$ D. R. Welch, D. V. Rose, B. V. Oliver, and R. E. Clark, Nucl. Instrum. Methods Phys. Res. A 464, 134 (2001).

${ }^{22}$ F. N. Beg, A. R. Bell, A. E. Dangor, C. N. Danson, A. P. Fews, M. E. Glinsky, B. A. Hammel, P. Lee, P. A. Norreys, and M. Tatarakis, Phys. Plasmas 4, 447 (1997).

${ }^{23}$ Y. T. Lee and R. M. More, Phys. Fluids 27, 1273 (1984).

${ }^{24}$ R. B. Stephens, Y. Aglitskiy, F. Amiranoff, C. Andersen, D. Batani, S. D. Baton, T. Cowan, R. R. Freeman, T. Hall, S. P. Hatchett, J. M. Hill, M. H. Key, J. A. King, J. A. Koch, M. Koenig, A. J. MacKinnon, K. L. Lancaster, E. Martinolli, P. Norreys, E. Perelli-Cippo, M. Rabec Le Gloahec, C. Rousseaux, J. J. Santos, F. Scianitti, and R. A. Snavely, Phys. Rev. E 69, 066414 (2004).

${ }^{25}$ P. E. Young, M. E. Foord, J. H. Hammer, W. L. Kruer, M. Tabak and S. C. Wilks, Phys. Rev. Lett. 75, 1082 (1995); K. A. Tanaka, H. Hashimoto, R. Kodama, K. Mima, Y. Sentoku, and K. Takahashi, Phys. Rev. E 60, 3283 (1999); K. Takahashi, R. Kodama, K. A. Tanaka, H. Hashimoto, Y. Kato, K. Mima, F. A. Weber, T. W. Barbee, Jr., and L. B. Da Silva, Phys. Rev. Lett. 84, 2405 (2000).

${ }^{26}$ K. A. Tanaka, private communication (2004)

${ }^{27}$ S. C. Wilks, A. B. Langdon, T. E. Cowan et al., Phys. Plasmas 8, 542 (2001); J. Denavit, Phys. Fluids 22, 1384 (1979).

${ }^{28}$ R. A. Snavely, M. H. Key, S. P. Hatchett et al., Phys. Rev. Lett. 85, 2945 (2000).

${ }^{29}$ P. K. Patel, A. J. MacKinnon, T. E. Cowan et al., Phys. Rev. Lett. 91, 125004 (2003).

${ }^{30}$ J. D. Bonlie, F. Patterson, D. Price, B. White, and P. Springer, Appl. Phys. B: Lasers Opt. 70, S155 (2000); F. Patterson, J. Bonlie, D. Price, B. White, and P. Springer, Ultrafast Optics 99 Conference, Ascona, Switzerland, 11-17 July 1999.

${ }^{31}$ M. Tabak, J. H. Hammer, E. M. Campbell et al., Lawrence Livermore 
National Laboratory patent disclosure, IL8826B, 1997, Lawrence Livermore National Laboratory, Livermore, CA; S. Hatchett and M. Tabak, "Cone focus geometry for Fast Ignition," presentation given at 30th Annual Anomalous Absorption Conference, Ocean City, MD (April 2000); S. Hatchett, M. Herrmann, M. Tabak et al., Bull. Am. Phys. Soc. 46, 47 (2001).

${ }^{32}$ R. Stephens, S. P. Hatchett, M. Tabak et al., Phys. Plasmas 12, 056312 (2004).

${ }^{33}$ Y. Sentoku, K. Mima, H. Ruhl, Y. Kodama, R. Kodama, and T. E. Cowan, Phys. Plasmas 11, 3083 (2004).

${ }^{34}$ R. P. J. Town, L. Cottrill, W. L. Kruer et al., "LSP calculations of electron transport experiments," International Workshop of Fast Ignition and High
Field Physics, Kyoto, 2004.

${ }^{35}$ R. Kodama, H. Azechi, H. Fujita et al., in Ref. 19 , p. 333.

${ }^{36}$ R. Kodama, P. A. Norreys, K. Mima et al., Nature (London) 412, 798 (2001); R. Kodama, H. Shiraga, K. Shigemori et al., ibid. 418, 933 (2001).

${ }^{37}$ N. Miyanaga, H. Azechi, K. A. Tanaka et al., in Ref. 19.

${ }^{38}$ Eye on Technology, OE Mag. 4, 8 (2004).

${ }^{39}$ C. P. J. Barty, M. Key, J. Britten et al., in Ref. 19.

${ }^{40}$ G. R. Bennett, O. L. Landen, R. F. Adams et al., Rev. Sci. Instrum. 74, 657 (2001) 